Medical Research Archives. Volume 5, issue 7. July 2017.

\title{
Microinvasive oral squamous cell carcinoma- A clinicopathological study
}

\section{Contributors:}

1) Dr. Sheeba Alex, MDS

Professor,

Dept. of Oral Pathology and Microbiology, Y.M.T Dental College and Hospital, Institutional area, Sector-4, Kharghar,

Navi Mumbai-410210,

Maharashtra, India

2) Dr. Prachi Ramchandra Bhandare, MDS Dept. of Oral Pathology and Microbiology, Y.M.T Dental College and Hospital, Institutional area, sector-4, Kharghar, Navi Mumbai410210, Maharashtra, India

3) Dr. Sangeeta R Patankar, MDS Professor and Head, Dept. of Oral Pathology and Microbiology, Y.M.T Dental College and Hospital, Institutional area, sector-4, Kharghar,

Navi Mumbai-410210,

Maharashtra, India

4) Dr. Gokul Sridharan, MDS Reader, Dept. of Oral Pathology and Microbiology, Y.M.T Dental College and Hospital, Institutional area, sector-4, Kharghar, Navi Mumbai410210, Maharashtra, India

\section{Corresponding author:}

Dr. Gokul Sridharan MDS

Reader, Dept. of oral Pathology and Microbiology YMT Dental College and Hospital, Kharghar, Navi

Mumbai-410210

E-mail: drgokuls@gmail.com

Source(s) of support: None

Conflicting Interest: None

\section{Abstract:}

Background and objectives: Microinvasive oral squamous cell carcinoma is an early stage malignancy characterized by invasion of superficial lamina propria without invasion of deeper structures. These lesions have a varied clinical presentation, minimal lymphatic involvement and a better prognosis. However, there is no clear consensus on its diagnosis and subsequent management. The aim of this study is to assess the depth of invasion of confirmed cases of microinvasive oral squamous cell carcinoma using special stains and immunohistochemistry with clinical correlation of these lesions.

Method: Tissue specimen of clinically diagnosed cases of oral leukoplakia $(n=90)$, oral submucous fibrosis $(n=60)$ and oral carcinoma $(n=50)$ were microscopically evaluated for the presence of microinvasion by two independent examiners. The next step involved confirming the basement membrane breach by using Periodic acid Schiff (PAS) stain for evaluating the integrity of basement membrane. The confirmed cases were assessed using special stain and immunohistochemistry to measure the depth of invasion and were further correlated with the clinical features.

Results: A total of 29 cases were confirmed as microinvasive OSCC. These included clinically diagnosed 11 cases of leukoplakia, 7 cases of OSMF and 11 cases of carcinoma. Median age at diagnosis was 46 yrs; males (69\%) were more affected than females $(31 \%)$. The predominant risk factor was tobacco chewing alone $(31.03 \%)$ which was in the form of gutkha and arecanut; common site was buccal mucosa (76\%); predominant clinical presentation was a patch; and histopathology revealed varying grades of epithelial dysplasia. The mean depth of invasion was $31.29 \mu \mathrm{m}$ $(0.03 \mathrm{~mm})$ with the range being $16.91-66.6 \mu \mathrm{m}(0.02-$ $0.07 \mathrm{~mm})$.

Conclusion: The study suggest that depth of invasion could be a reliable marker to assess microinvasion and that severity of epithelial dysplasia is not a pre-requisite for microinvasive carcinoma.

Keywords: Microinvasion; Oral squamous cell carcinoma; Epithelial dysplasia; Depth of invasion; Oral leukoplakia; Potentially malignant disorders 
Medical Research Archives. Volume 5, issue 7. July 2017.

\section{Microinvasive oral squamous cell carcinoma- A clinicopathological study}

\section{Introduction:}

Oral cancer is a significant disease affecting humans and squamous cell carcinoma (OSCC) accounts for over $90 \%$ of the reported malignancies of the oral cavity. OSCC is one of the most common epithelial malignancies with significance morbidity and mortality and possess a multifactorial etiology. Factors such as habits, genetic, environmental, their interactions with each other and viral factors have been implicated in the etiopathological continuum of oral cancer.

${ }^{2}$ Despite diagnostic and therapeutic advances over the decades, the disease remains a challenge for medical professionals with the five-year survival rate being around $45 \%$. $^{3}$ The mortality rate associated with oral cancer is high because it is routinely discovered late, commonly after metastasis to lymph nodes or neck has already occurred. ${ }^{4}$ While OSCC may arise de novo, most of them are preceded by the presence of clinically visible changes of oral mucosa which have a potential for malignant transformation. These lesions are together categorized as potentially malignant disorders. Leukoplakia and erythroplakia are the most commonly encountered disorders while OSMF, lichen planus and discoid lupus erythematosus are less likely to show malignant changes. ${ }^{5}$ The incidence rate of potentially malignant disorders ranges between $0.6 / 1000$ to $30.2 / 1000$ and the worldwide prevalence is $0.2 \%$ to $11.3 \%$. ${ }^{6}$ Leukoplakia is the most commonly encountered potentially malignant disorder of the oral cavity with a malignant transformation rate ranging from $0.1 \%$ to 17.5\%. ${ }^{7}$ The clinical appearance of these lesions may vary from being innocuous to sinister and hence needs histopathological assessment for confirmation of diagnosis and progression to malignancy.

The final diagnosis of clinical suspicious malignancy depends on the histopathological examination of the involved tissue. Histological grading is an important tool which helps in predicting the clinical and biological behavior of OSCC. The first histological grading system was initiated by Broder based on the degree of differentiation and keratinization of tumor cells and categorized as Grade I- well differentiated tumors $(75-100 \%$ cells are differentiated); Grade II-moderately differentiated tumors (50-75\% cells are differentiated); Grade IIIpoorly differentiated tumors $(25-50 \%$ cells are differentiated) and Grade IV- anaplastic tumor $\left(0-25 \%\right.$ cells are differentiated). ${ }^{8}$ While, molecular advancements and its applications have significantly contributed to our understanding and diagnosis of head and neck carcinogenesis, basic histological assessment of the tumor tissue and its grading continues to play a pivotal role in routine clinical practice.

One aspect of OSCC that requires consideration is the microinvasive squamous cell carcinoma which is an early stage relatively 'thin' tumor without invasion of deep tissues. 9, 10 There is no clarity on defining microinvasive oral squamous cell carcinoma although classification systems do exist for similar lesions occurring elsewhere, viz. microinvasion in cervical cancer. Microinvasive OSCC is an early stage relatively thin tumor confined to the papillary lamina propria as defined by the depth of the rete process. ${ }^{11}$ The depth generally varies from $0.5-2 \mathrm{~mm}$ and must be measured from the adjacent non-neoplastic surface epithelium because of greater variations in epithelial thickness. ${ }^{12}$ Microinvasion is also defined as one with an irregular infiltrative border often accompanied by a reactive desmoplasia and not by a pushing type expansion of hyperplastic epithelium. ${ }^{13}$

Microinvasive carcinoma is a biologically malignant lesion potentially capable of gaining access to lymphatics and vascular channels. ${ }^{14}$ Even though majority of lesions with microinvasion have a better prognosis, it is prudent to assess the depth of invasion as 
Medical Research Archives. Volume 5, issue 7. July 2017.

\section{Microinvasive oral squamous cell carcinoma- A clinicopathological study}

proximity to blood vessels and lymphatics increases the risk of nodal metastasis. 'Depth of invasion' refers to the extent of cancer growth into the tissue beneath an epithelial surface. ${ }^{15}$ Although measuring the depth is subjective in nature especially in areas of absence of basement membrane, it serves well in oral cancer owing to the varying epithelial thickness in different sites of the oral mucosa.

To date, there is minimal literature data describing the clinical appearance of microinvasive OSCC. On this basis, the objective of the study was to histologically analyze cases of microinvasive carcinoma with clinical correlation and to determine the utility of depth of invasion as a predictor for microinvasive oral squamous cell carcinoma.

\section{Materials and Method:}

Hematoxylin and eosin stained incisional biopsies of clinically suspected leukoplakia (n =90), oral submucous fibrosis (OSMF) ( $\mathrm{n}=$ $60)$ and carcinoma of oral cavity $(n=50)$ were retrospectively evaluated by two independent examiners for presence of microinvasion. Inclusion criteria to consider the presence of microinvasion consisted of lesions with suspected basement membrane breach and presence of dense inflammatory infiltrate while cases that were specifically graded based on Broder's criteria, and lesions with ulcerative epithelium were not included. Lesions with ulcerative epithelium do not provide accurate representation of basement membrane breach thus giving rise to false positive results and hence excluded. The histopathologic criteria followed to suspect basement membrane breach by both the examiners included one or more of the following: presence of ragged borders, single 'dropping off' of cells and presence of dense inflammatory infiltrate. Independent observation of all selected cases was done by both the examiners. In cases where there was mismatch in final interpretation, slides were seen together by both examiners and a final decision was made.

Based on the observations, 18 cases of clinical leukoplakia, 11 cases of clinical OSMF and 12 cases of clinical carcinoma were considered as suspected cases of microinvasion on microscopic examination. The next step involved confirming the basement membrane breach by using periodic acid schiff (PAS) stain for evaluating the integrity of basement membrane. PAS staining was done using standard protocol and the stained slides were examined by the same two independent examiners. Doubtful interpretation was mutually resolved by both the examiners. On completion of the following, a total of 29 cases were finally considered as microinvasive OSCC. These included clinically diagnosed 11 cases of leukoplakia, 7 cases of OSMF and 11 cases of carcinoma. The cases selected as microinvasive OSCC were further subjected to two analyses.

a. Clinical data collected included age, sex, site, the clinical appearance of the lesions, tobacco and alcohol habits (smoking or smokeless tobacco or both, the type of tobacco usage, the presence or absence of alcohol consumption) and lymph node status.

b. IHC staining was done using anticytokeratin, Pan (C11) antibody to stain the 'invaded' epithelial cells. This antibody stains cytokeratins 4, 5, 6, 10, 13 which are highly expressed in the basal and supra-basal cells, helps to differentiate the invaded postitively stained epithelial cells from mesenchymal cells and further aid in measurement of depth of invasion.

\subsection{Depth of invasion}

The depth of invasion was measured from the basement membrane or in areas of basement membrane loss, from an imaginary line reconstructing the basement membrane from 
Medical Research Archives. Volume 5, issue 7. July 2017.

\section{Microinvasive oral squamous cell carcinoma- A clinicopathological study}

the adjacent epithelium to the deepest point of invasion in connective tissue using motic advanced images optical micrometer 3.2 software calibrated to $150 \mu \mathrm{m}$ for a $40 \mathrm{x}$ objective lens as prescribed by the manufacturer. The point of deepest invasion of the tumor epithelial cells was identified in a $4 \mu \mathrm{m}$ thick section of the IHC stained slide and the depth was measured and recorded.

\section{Results:}

In total, 29 cases were confirmed as microinvasive OSCC from all the reported cases of leukoplakia, OSMF and carcinoma in the department of Oral Pathology and Microbiology of our institution. The median age and range at the time of diagnosis for oral leukoplakia, OSMF and OSCC was 55years (24-76yrs), 38 yrs (24-51yrs) and 56yrs (25$75 \mathrm{yrs})$ respectively. Figure 1 describes the box plot for the age distribution among the three groups. Age distribution was almost equal in age group of 20-40 yrs, 41-60 yrs and $>60 y \mathrm{rs}(34.5 \%, 31.03 \%$ and $34.5 \%$ respectively) (Table 1). Gender distribution was favourable to males $(69 \%)$ as compared to females (31\%) (Table 1 and Figure 2). The predominant risk factor was tobacco chewing alone $(31.03 \%)$ which was in the form of gutkha and arecanut while the combination of tobacco smoking and chewing tobacco and alcohol was comparatively lesser. This is a common trend in countries like India where tobacco chewing is a primary risk factor for oral cancer. ${ }^{[16]}$ The observed lesions that were histologically proven to be microinvasive OSCC were frequently noted in buccal mucosa (76\% of the lesions) which was similar to data obtained from countries like India where alveolo-gingivo-buccal sulcus is the common site. ${ }^{[17]}$

The predominant clinical appearance of the lesions diagnosed as microinvasion was that of a patch $(48 \%, \mathrm{n}=22)$ followed by ulcer $(35 \%, \mathrm{n}=10)$ and growth $(17 \%, \mathrm{n}=5)$ (Table 2 ). Of the 29 cases, lymph nodes were not palpable in 21 cases $(72 \%)$ while in 8 cases (28\%) of the cases lymphnodes were palpable. Majority of the patients were symptomatic (pain, burning sensation and tenderness) and were aware of the presence of the lesion. On evaluation of the status of dysplasia among the confirmed cases of microinvasion, it was observed that 2 cases had no dysplastic changes, 14 cases showed mild dysplasia, 6 were of moderate dysplasia, 5 cases showed severe dysplasia and 2 were carcinoma in situ.

The measurement of depth of invasion revealed that the mean depth of invasion was $31.29 \mu \mathrm{m}(0.03 \mathrm{~mm})$ with the range being $16.91-66.6 \mu \mathrm{m}(0.02-0.07 \mathrm{~mm})$. The deepest point of invasion amongst all the cases was $66.6 \mu \mathrm{m}(0.07 \mathrm{~mm})$ (Table 3). Figure 3 labels the photomicrograph (magnification- $\mathrm{x} 400$ ) showing microinvasion in (A): hematoxylin and eosin staining (arrow), (B): periodic acid Schiff staining (arrow), (C): immunohistochemical staining (arrow)

\section{Discussion:}

The data obtained in the present study was similar to the results of Pentenero $\mathrm{M}$ et al (2011) ${ }^{10}$ as well as of O- Charoenrat et al (2003) ${ }^{18}$ and Gandolfo et al (2006) ${ }^{19}$. Analysis of OSCC cases by Pires et al (2013) ${ }^{20}$ identified microinvasive carcinoma in $7.5 \%$ of their study sample. Their findings included equal distribution amongst males and females with a mean age of 67.2 years with most lesions showing leukoerythroplakic areas (73.1\%), and commonly affecting the border of the tongue ${ }^{20}$. Clinical appearance of the lesions in our study demonstrated that a patch (49\%) was the predominant pattern of presentation with ulcer and growth being the other types. This data emphasizes the fact that despite the invasive nature, the clinical appearance more often resembles that of a potentially malignant disorder/ non-invasive lesion. Assessment of the lymph node status 
Medical Research Archives. Volume 5, issue 7. July 2017.

\section{Microinvasive oral squamous cell carcinoma- A clinicopathological study}

of the cases included in the study revealed that there was no lymph node involvement in majority of cases suggesting minimal lymphatic spread in microinvasive carcinoma.

The measured depth of invasion in our study ranged from $16.91-66.6 \mu \mathrm{m}(0.02-0.07$ $\mathrm{mm})$ with an average of $31.29 \mu \mathrm{m}(0.03 \mathrm{~mm})$. The invaded cells were almost always confined to the papillary lamina propria without deep invasion. This may suggest a favourable prognosis thereby aiding in a better treatment plan with decreased postoperative morbidity. The results depth of invasion obtained in our study are in accordance with the study done by Haberland et al (2012) ${ }^{21}$ in which there was focal and/ or superficial invasion of less than $2 \mathrm{~mm}$ and no deeper than the lamina propria in microinvasive OSCC.

Microinvasive carcinoma is an entity described in depth in sites such as larynx, cervix and breast. For laryngeal lesions, microinvasive cancer includes the presence of scattered malignant cells within the submucosa just below the basement membrane or within 1-2 $\mathrm{mm}$ of basement membrane. ${ }^{22}$ Barnes (2001) defined microinvasive carcinoma of larynx as invasive SCC that extends into the stroma by $\leq 0.5 \mathrm{~mm}$, as measured from adjacent epithelial basement membrane. 23 Microinvasive carcinoma of the cervix is described based on the sub-division of stage I cancers as a lesion which invades the cervical stroma to the depth of $3 \mathrm{~mm}$ below the base of the epithelium and no more than $7 \mathrm{~mm}$ wide. ${ }^{24}$ Microinvasive carcinoma of breast is a term used to describe a borderline difference between completely contained ductal carcinoma in situ and a minimally invasive ductal carcinoma. A very small amount of malignant cells are found beyond the duct ${ }_{25}$ lining and into the surrounding stromal tissue.
However, currently there is no uniform definition among pathologists regarding microinvasive oral squamous cell carcinoma. This could be attributed to the lack of a welldefined grading system, limited literature data and the variable timing and mode of clinical presentation. There are no objective criteria for its diagnosis and it usually depends on the pathologists' judgment of the lesion. This is further hampered by the dense inflammation and the plane of section through bulbous or branching rete pegs. ${ }^{26}$ Also, no universally accepted guidelines exist for treatment of clinically negative nodes in early stage OSCC and there is continuing debate on employing elective neck dissection for treatment of the same. $^{27}$

Histologically, microinvasive carcinoma can occur in 2 unrelated phases. The first is the development from and a continuum of carcinoma in situ. The second is invasion from an epithelium demonstrating no evidence of carcinoma in situ. ${ }^{22}$ This is an important aspect as can be seen from our study, the results of which reveal that a higher dysplastic grade is not a pre-requisite for invasion and that invasion can occur even in the presence of minimal dysplasia of the oral epithelium.

In cases of microinvasive OSCC, it is prudent to assess the depth of invasion and its relationship to blood vessels and other stromal components which contributes to invasion and metastasis. This study evaluated the depth of invasion of the tumor cells into the underlying stroma. The relevance of estimating depth of invasion is in regard to proximity to blood vessels and lymphatics which determines the risk of nodal metastases as close proximity to the stromal components facilitates the tumor's ability to disseminate. 12

Depth of invasion is the extent of cancer growth into the tissue beneath an epithelial surface. Although depth of invasion 
Medical Research Archives. Volume 5, issue 7. July 2017.

\section{Microinvasive oral squamous cell carcinoma- A clinicopathological study}

evaluation is subjective in nature, it provides an accurate assessment of invasive front beneath the basement membrane unlike tumor thickness which refers to the entire tumor mass. Tumor thickness might result in an inaccurate measurement owing to the varying thickness of oral epithelium at different sites and hence depth of invasion was chosen as a parameter. ${ }^{10}$

Diagnosis of microinvasive OSCC needs careful evaluation of the entire tissue section and islands of epithelium within the lamina propria should be regarded with suspicion although it is necessary to rule out sectioned rete process which can be confirmed by the presence of cellular atypia in the invaded epithelial component. In the present study, microinvasion was suspected based on the presence of irregular epithelium-connective tissue interface, invaded epithelial cells and dense inflammatory infiltrate in underlying connective tissue. ${ }^{28}$ However, difficulties may arise when the invasion is very minimal and is masked by the presence of dense inflammation. It then becomes necessary for the pathologist to identify the basement membrane breach which may not be always possible in routine $\mathrm{H}$ and $\mathrm{E}$ stained sections. In such cases, the use of histochemical techniques can function as useful adjuvants in confirming the diagnosis.

In conclusion, the current grading system does not have any provision for the categorization of microinvasive OSCC and the treatment of clinically unsuspicious lesion and negative nodes has been a matter of continuous debate. Therefore, guidelines for the diagnosis and management of microinvasive OSCC as a separate entity is mandatory to avoid injudicious radical therapy. Soon, the diagnostic procedure should also benefit from the advances in molecular methods which must be translated to predict the behavior of all tumors classified as microinvasive OSCC that can progress to advanced grades of carcinoma. 
Medical Research Archives. Volume 5, issue 7. July 2017.

\section{Microinvasive oral squamous cell carcinoma- A clinicopathological study}

\section{References:}

1. Rao SVK, Mejia G, Thomson KR, Logan $R$. Epidemiology of oral cancer in Asia in the past decade- An update (2000-2012). Asian Pac J Cancer Prev 2013; 14:556777

2. Mehrotra R, Yadav S. Oral squamous cell carcinoma: etiology, pathogenesis and prognostic value of genomic alterations. Ind J Cancer 2006; 43: 60-6

3. Larsen SR, Johansen J, Sorensen JA. The prognostic significance of histological features in oral squamous cell carcinoma. J Oral Pathol Med 2009; 38:657-62

4. Sugimoto M, Wong DT, Hirayama A, Soga T, Tomita M. Capillary electrophoresis mass spectrometry-based saliva metabolomics identified oral, breast and pancreatic cancer specific profiles. Metabolomics 2010; 6:78-95.

5. Brennan M, Migliorati CA, Lockhart PB, Wray D, Al-Hashimi I, Axell T, Bruce AJ, Carpenter W, Eisenberg E, Epstein JB. Management of oral epithelial dysplasia: a review. Oral Surg Oral Med Oral Pathol Oral radiol Endod 2007; 103:S19.e1S19.e12.

6. Dionne KR, Warnakulasuriya S, Zain RB, Cheong SC. Potentially malignant disorders of the oral cavity: Current practice and future directions in the clinic and laboratory. Int $\mathbf{J}$ Cancer 2015; 136:503-15.

7. Van der Waal I. Oral potentially malignant disorders: Is malignant transformation predictable and preventable? Med Oral Patol Oral Cir Bucal 2014; 19:e386-e390.
8. Broders AC. Squamous cell epithelioma of skin: A study of 256 cases. Annals of surgery $1921 ; 2: 141-60$

9. Shankar AA, Gokul. S. The dilemma of microinvasion. Head neck oncol 2014; 6:30

10. Pentenero M, Narone R, Motta F, Marino R, Gassino L, Broccoletti R, et al. Clinical features of microinvasive stage I oral carcinoma. Oral Dis 2011; 17:298-303.

11. Heffner DK. Lets make grading of squamous cell carcinomas more meaningful to clinicians (via "Ed's Insight"). Ann Diagn Pathol 2002; 6:399403.

12. Woolgar JA, Triantafyllon A. Pitfalls and procedures in the histopathological diagnosis of oral and oropharyngeal SCC: A review of the role of pathology in prognosis. Oral Oncol 2009; 45:361-85.

13. Cardesa A. Slootweg PJ. Benign and potentially malignant lesions of squamous epithelium. In: Pathology of head and neck, Berlin: Springer 2006; 13

14. Batsakis JG. Clinical pathology of oral cancer. In Shah JP, Johnson NW, Batsakis JG. Oral Cancer. $1^{\text {st }}$ ed. Martin Dunitz, 2003: 75-129.

15. Wenig BM. Squamous cell carcinoma of the upper aerodigestive tract: Precursors and problematic variants. Mod Pathol 2002; 15:229-54.

16. Bagan. J, Sarrion. G and Jimmenez Y. Oral Cancer: Clinical features. Oral Oncol 2010; 46: 414-417.

17. Johnson NW, Jayasekara P, Amarasinghe AA. Squamous cell carcinoma and precursor lesions of the oral cavity: 
Medical Research Archives. Volume 5, issue 7. July 2017.

\section{Microinvasive oral squamous cell carcinoma- A clinicopathological study}

epidemiology and etiology. Periodontology 2000. 2011; 57: 19-37.

18. O- Charoeurat P, Pillai G, Patel S, Fishcer C, Archer D, Eccles S, et al. Tumor thickness predicts cervical node metastases and survival in early oral tongue cancer. Oral Oncol 2003; 39: 386390.

19. Gandolfo S, Scully C, Carrozzo M. Oral Medicine. Churchill LivingstoneElsevier: Philadelphia 2006.

20. Pires FR, Ramos AB, de Oliveira JBC, Tavares AS, da Luz PSR, dos Santos TCRB. Oral squamous cell carcinoma: clinicopathological features from 346 cases from a single oral pathology service during an 8-year period. J Appl Oral Sci 2013; 21: 460-7.

21. Haberland C, Sasaki C, Judson B, Virk R, Prasad M. Superficially invasive squamous cell carcinoma of the oral cavity; Oral Surg Oral Med Oral Radiol Oral Pathol 2012; e56.

22. Wenig BM. Squamous cell carcinoma of the upper aerodigestive tract: Precursors and problematic variants. Mod Pathol 2002; 15: 229-254.
23. Barnes L. Diseases of the larynx, hypopharynx and esophagus. In: Barnes L, editor. Surgical pathology of the head and neck. New York: Dekker; 2001; 127237.

24. Ziraljevic M, Vujkor T, Nincic D, Mandic A, Zikic D, Masrilovic K. Arch Oncol 2004; 12: 142-144.

25. Padmore RF, Fowble B, Hoffman J, Rosser C, Hanlon A, Parchofsky AS. Microinvasive breast carcinoma: Clinicopathologic analysis of a single institution experience. Cancer 2000; 88: 1403-1409.

26. Speight P. Update on oral epithelial dysplasia and progression to cancer. Head and Neck Pathol 2007; 1:61-66.

27. Yuen AP, Ho CM, Chow TL, Tang LC, Cheung WY, Ng RW, et al. Prospective randomized study of selective neck dissection versus observation for NO neck of early tongue carcinoma. Head Neck 2009; 31: 765-72.

28. Mashberg A, Samit A. Early diagnosis of asymptomatic oral and oropharyngeal squamous cancers. CA cancer $\mathrm{J}$ Clin 1995; 45: 328-51. 
Medical Research Archives. Volume 5, issue 7. July 2017.

Microinvasive oral squamous cell carcinoma- A clinicopathological study

\section{Figures:}

Figure 1: Box plot showing the age distribution in oral leukoplakia, OSMF and OSCC in the study participants

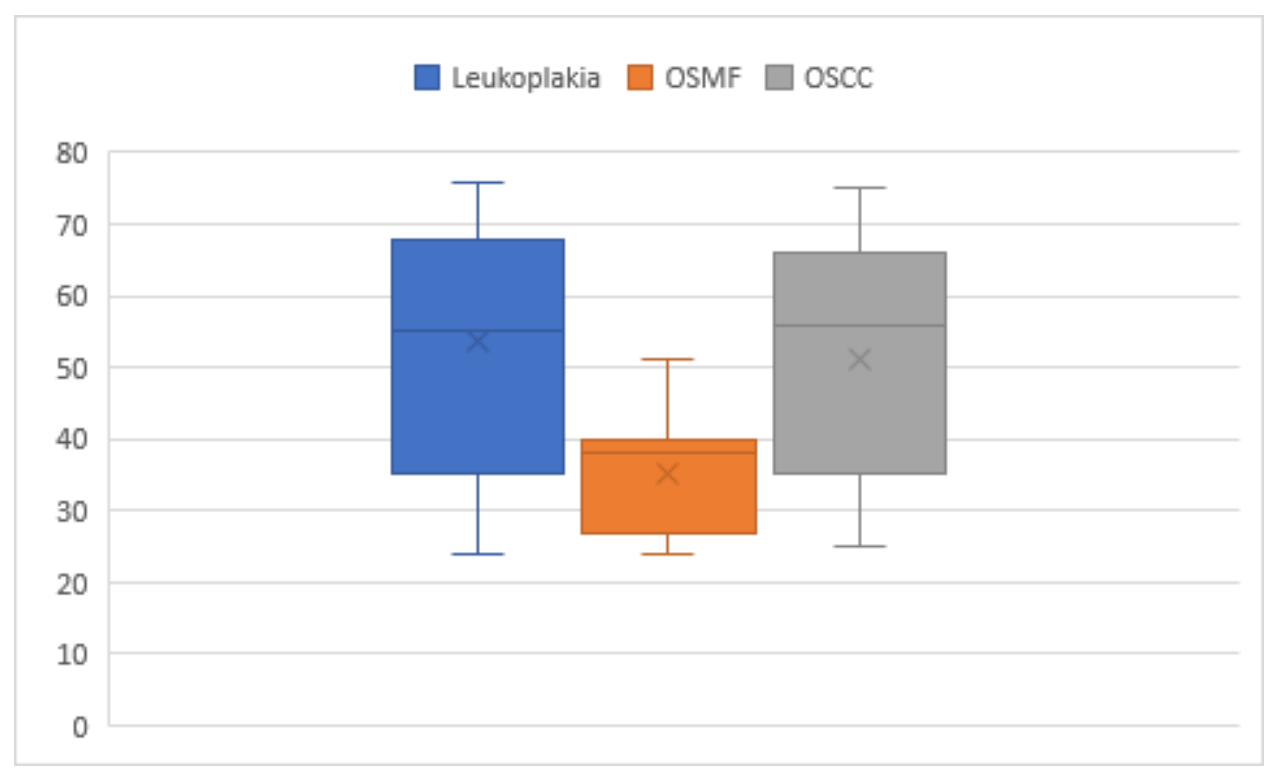

Figure 2: Gender distribution of the confirmed microinvasive OSCC cases in the study participants

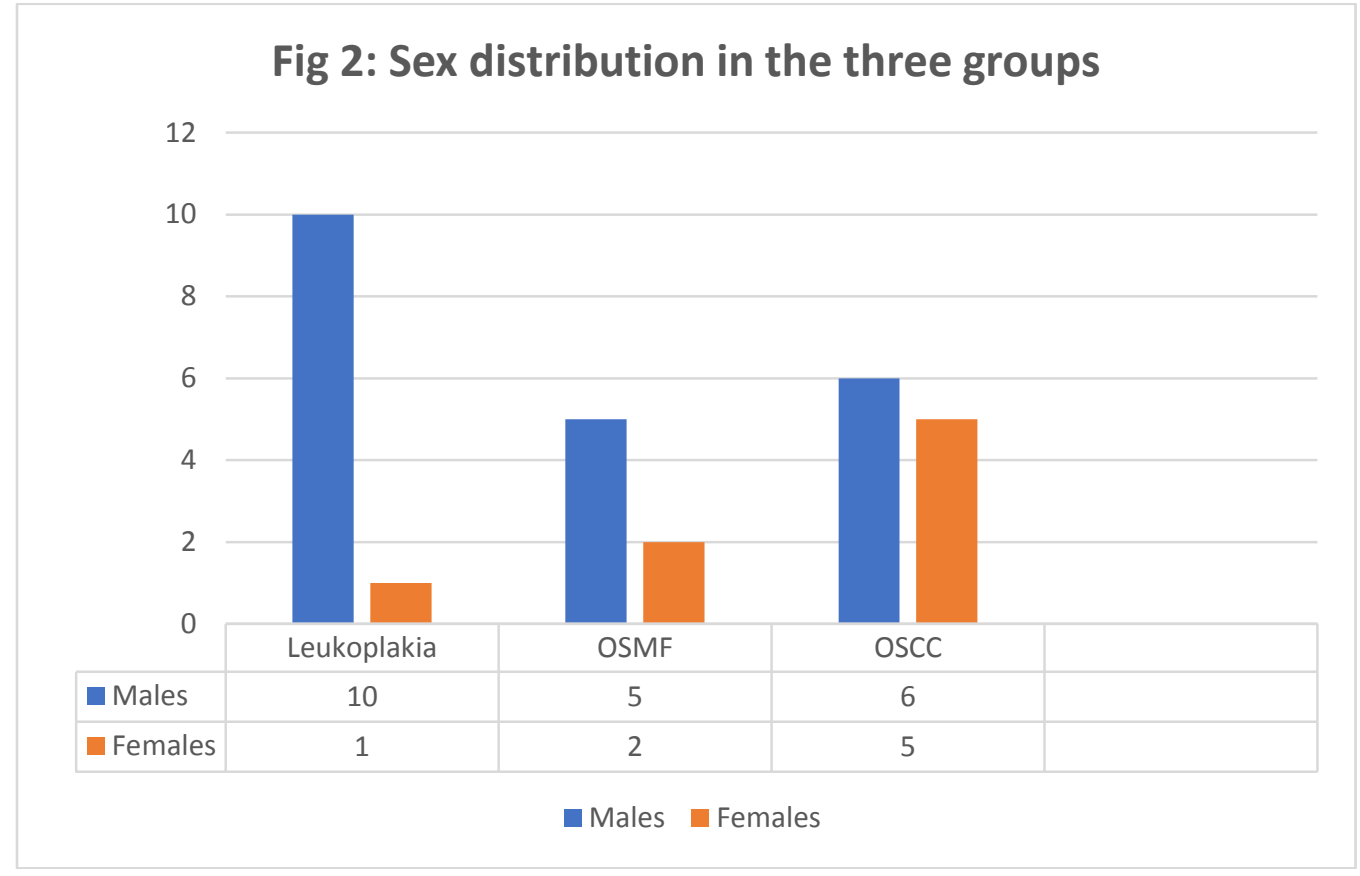


Medical Research Archives. Volume 5, issue 7. July 2017.

\section{Microinvasive oral squamous cell carcinoma- A clinicopathological study}

Figure 3: Photomicrograph (magnification- $x$ 400) showing microinvasion in (A): hematoxylin and eosin staining (arrow), (B): periodic acid Schiff staining (arrow), (C): immunohistochemical staining (arrow)
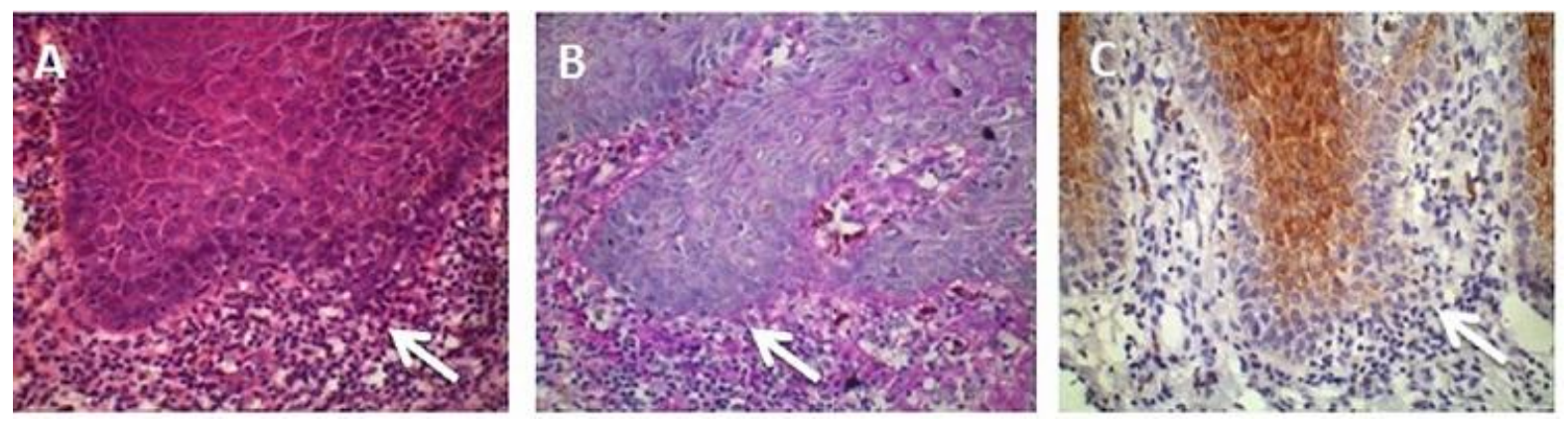
Medical Research Archives. Volume 5, issue 7. July 2017.

Microinvasive oral squamous cell carcinoma- A clinicopathological study

\section{Tables:}

Table 1: Age and sex distribution of the confirmed microinvasive OSCC cases

\begin{tabular}{|c|c|c|c|c|c|}
\hline Age & Number & Percentage (\%) & Sex & Number & Percentage (\%) \\
\hline $\begin{array}{c}20-40 \\
\text { years }\end{array}$ & 10 & 34.48 & Male & 20 & 69 \\
\hline $\begin{array}{c}41-60 \\
\text { years }\end{array}$ & 9 & 31.03 & Female & 9 & 31 \\
\hline $\begin{array}{c}>60 \\
\text { years }\end{array}$ & 10 & 34.48 & & & \\
\hline
\end{tabular}

Table 2: Site of lesion and clinical appearance

\begin{tabular}{|c|c|c|c|c|c|}
\hline Site & Number & Percentage (\%) & Appearance & Number & Percentage (\%) \\
\hline Buccal mucosa & 22 & 75.86 & Patch & 14 & 48.28 \\
\hline Vestibule & 4 & 13.79 & Growth & 5 & 17.24 \\
\hline Tongue & 1 & 3.45 & & & \\
\hline Floor of mouth & 1 & 3.45 & Ulcer & 10 & 34.48 \\
\hline Commisure & 1 & 3.45 & & & \\
\hline
\end{tabular}

Table 3: Depth of invasion

\begin{tabular}{|c|c|c|c|}
\hline & Mean \pm SD & Range & $\begin{array}{c}\text { Deepest point of } \\
\text { invasion }\end{array}$ \\
\hline $\mathrm{n}=29$ & $\begin{array}{c}31.29 \pm 13.69 \mu \mathrm{m} \\
(0.03 \pm 0.01 \mathrm{~mm})\end{array}$ & $\begin{array}{c}16.31-66.60 \mu \mathrm{m} \\
(0.02-0.07 \mathrm{~mm})\end{array}$ & $\begin{array}{c}66.60 \mu \mathrm{m} \\
(0.07 \mathrm{~mm})\end{array}$ \\
\hline
\end{tabular}

